\title{
The data do not seem to support a benefit to BCAA supplementation during periods of caloric restriction
}

\author{
Brad P. Dieter ${ }^{1}$, Brad Jon Schoenfeld ${ }^{2^{*}}$ and Alan A. Aragon ${ }^{3}$
}

\begin{abstract}
$J$ Int Soc Sports Nutr 13:1-015-0112-9, 2016 describe the efficacy of branched chain amino acid (BCAA) supplementation and resistance training for maintaining lean body mass during a calorie-restricted diet, and claim that this occurs with concurrent losses in fat mass. However, the reported results appear to be at odds with the data presented on changes in fat mass. This letter discusses the issues with the paper.
\end{abstract}

\section{To the Editor,}

Dudgeon et al. [1] describe the efficacy of branched chain amino acid (BCAA) supplementation and resistance training for maintaining lean body mass during a calorie-restricted diet, and claim that this occurs with concurrent losses in fat mass. However, the reported results appear to be at odds with the data presented on changes in fat mass.

The study reports a statistically significant change in fat mass for the group supplementing with BCAAs, but not in the placebo (isocalorically matched carbohydrate [CHO] beverage) group. However, this outcome is paradoxical with the results. Table 2 states that the BCAA group lost $0.6 \mathrm{~kg}$ of fat mass while the $\mathrm{CHO}$ group lost $1.4 \mathrm{~kg}$. Given that the standard errors were virtually identical between groups (the SE was actually less for the $\mathrm{CHO}$ group pre-study), it is counterintuitive to believe that statistical probability for a true effect would be higher in the group that supplemented with BCAA.

If the results were in fact correctly reported with respect to statistical probability, then this appears to be a case of using the wrong statistical measures to analyze the data. The authors quizzically chose a combination of paired and unpaired t-tests for analysis, which is prone to bias based on the uniformity of the direction of

\footnotetext{
* Correspondence: brad@workout911.com

${ }^{2}$ Department of Health Sciences, Lehman College, Bronx NY, USA

Full list of author information is available at the end of the article
}

change for each subject. Indeed, multiple t-tests heighten the probability of making at least one type one error, and the increased error rate may be substantial [2]. The appropriate statistical measures that should have been employed include repeated measures ANOVA, repeated measures mixed model, or perhaps an ANCOVA on the change scores. Moreover, a priori alpha values alone provide an incomplete picture of the importance of results [3]. Magnitude-based statistics such as effect size (ES) are necessary to provide a more comprehensive perspective of the relative meaningfulness of the results and thus draw appropriate practical implications from findings [4]. A computation of Cohen's D based on the data shows that the ES for change in fat mass for $\mathrm{CHO}$ condition was strong (0.81) while that of the BCAA condition was weak (0.29). In addition, given the small sample size it would have been beneficial to present the individual data points to show changes in fat mass and lean mass over the course of the study instead of the aggregate data. Lastly, given the discordance between statistical significance and physiological meaningfulness power calculations ought to have been conducted to indicate that the failure to reject the null hypothesis in the $\mathrm{CHO}$ group may have been a type II error.

Issues with interpreting results are further confounded by inconsistencies in the reported data. In the abstract and the results section the authors indicate that the BCAA group lost $0.05 \mathrm{~kg} \pm 0.08 \mathrm{~kg}$. We were unable to locate this data anywhere else in the manuscript. Based upon the data in Fig. 1 (Fig. 4 from the original paper), it 
Table $\mathbf{2}$ Changes in body mass variables before and after 8 week study period

\begin{tabular}{llllll}
\hline & Age $(\mathrm{yrs})$ & Height $(\mathrm{cm})$ & Body Mass $(\mathrm{kg})$ & Lean Mass $(\mathrm{kg})$ & Fat Mass $(\mathrm{kg})$ \\
\hline BCAA & $24.7 \pm 0.6$ & $177.9 \pm 4.6$ & $84.3 \pm 5.2$ & $72.2 \pm 4.7$ & $12.2 \pm 0.7$ \\
& & $84.2 \pm 4.8$ & $72.6 \pm 4.3$ & $11.6 \pm 0.7^{\mathrm{a}}$ \\
$\mathrm{CHO}$ & $23.5 \pm 0.6$ & $176.6 \pm 5.6$ & $78.3 \pm 2.9$ & $67.8 \pm 2.5$ & $105 \pm 0.5$ \\
& & & $76.0 \pm 2.4^{\mathrm{a}}$ & $66.9 \pm 2.5^{\mathrm{a}}$ & $9.1 \pm 0.7$ \\
\hline
\end{tabular}

denotes significant difference $(p<0.05)$ within BCAA and $\mathrm{CHO}$

All subjects were prescribed the same hypocaloric diet and exercise programs. The BCAA group received $28 \mathrm{~g}$ of BCAA (14 g prior/during each workout and $14 \mathrm{~g}$ post workout) while the $\mathrm{CHO}$ group received $28 \mathrm{~g}$ of a carbohydrate/electrolyte supplement (14 g prior/during each workout and $14 \mathrm{~g}$ post workout)

appears that the mean decrease in fat mass was actually $0.5 \%$, raising the possibility of an error in transcription. Another glaring issue with the data are the standard error bars, which do not seem to match the data in the text. For example, the BCAA group appears to be roughly $0.4 \mathrm{~kg}$, instead of the $0.08 \mathrm{~kg}$ reported in the text and the $0.08 \mathrm{~kg}$ put forth in the abstract. These discrepancies call into question the veracity of the study.

Finally, the data presented in the paper appear to be internally inconsistent. The resting metabolic rate (RMR) dropped significantly in the BCAA group (412 kcal/day) but not in the CHO group (no data presented). In the discussion, the authors quote, "The amount of lean tissue mass is essential in determining metabolic rate, where a greater amount of lean tissue increases RMR." Given the maintenance of lean body mass and the loss of fat mass in the BCAA group, and the loss of lean body mass and the apparent loss of fat mass in the $\mathrm{CHO}$ group, the RMR should have decreased in the $\mathrm{CHO}$ group but not the BCAA group. These phenomena ought to have been explained by the authors as it conflicts with the reported outcome of maintenance of lean body mass and greater fat loss in the BCAA group while the $\mathrm{CHO}$ group lost lean body mass.
When attempting to extrapolate the findings into practice, it would appear that changes in body composition are a product of the magnitude of weight loss as opposed to the supplementation protocol. Specifically, the greater preservation of LBM in the experimental condition can be attributed to the minimal loss of body fat in these subjects, not consumption of BCAA. On the other hand, the control group lost substantially more weight, so it would seem logical that they would not have retained LBM as well. The alternative hypothesis posed by the authors is not consistent with the current body of literature. As noted in a recent review by Morton et al. [5], there is a paucity of evidence supporting a beneficial effect for BCAA supplementation in promoting increases in muscle protein synthesis or lean mass, and in fact there might be a detrimental impact given that the AAs appear to antagonize each other in terms of transport both into circulation and likely into the muscle.

\section{Competing interests}

The authors declare that they have no competing interests.

\section{Authors' contributions}

All authors drafted the text, read and approved the final manuscript.

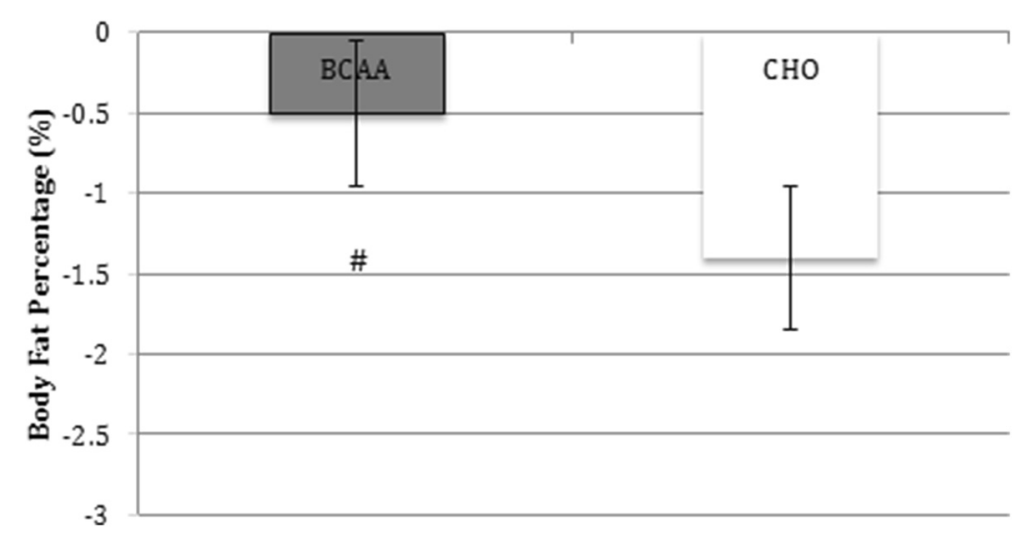

Fig. 1 Fat Mass Change 


\section{Authors' response}

Dieter et al.

The authors would like to thank JISSN for the opportunity to respond to the referenced communication and we appreciate the critical review of our work by Schoenfeld et. el. In the correspondence we will address what we feel to be the primary issues raised by Schoenfeld et. al.

First, in regards to which statistical method was used, the traditional mechanism for testing a pre vs. post treatment is $t$-test (paired for within groups and unpaired for between groups). Choosing a statistical methodology, regardless of type, will lend itself to either Type I or Type II error. This is not a debatable point. In choosing to answer our research question, "Does BCAA supplementation impact lean mass while on a hypocaloric diet?" we chose, and the JISSN editors and reviewers each independently agreed, a proven statistical model that has been used innumerable times in the literature when pre vs. post analysis of a treatment is examined.

Next, we have chosen to include a spaghetti graph of the body composition data, both lean tissue mass and fat mass, to allow the readers to see how each of the individuals responded during our intervention. As can be seen in Fig. 2 (Fig. 3 from the original paper), all control (CHO) subjects lost lean tissue mass, while in the BCAA group three gained and six lost lean tissue mass. Concerning fat mass, four CON subjects gained and four CON subjects lost, while all the BCAA group lost fat mass. (See Fig. 3
(Fig. 2 from the original paper)) Further, it should be clarified that Fig. 1 (Fig. 4 from the original paper) in the initial manuscript should have been labeled "Change in Percent Body Fat" not "Fat Mass Change." The corrected graph can be seen below (Fig. 4 (Fig. 1 from the original paper)).

Another issue raised by Schoenfeld et. al. was the lack of a change in RMR when changes were found in body composition. The variability in the data did not allow for significant changes in RMR to be found. As noted in our manuscript, we acknowledge that this did not fit given the changes detected in body mass. If more subjects were to have been involved in the study, perhaps a clearer picture of the impact of BCAA on RMR would have been found.

Finally, all body weight changes are relative to the individual, thus the comparison within groups and not just between. The impact of a hypocaloric diet is being ignored in the correspondence and it should not be since this, as noted in the title, is what we feel makes this a significant contribution to the body of scientific literature. Heavy resistance training without adequate calories to maintain lean mass will inevitably send the body into a catabolic state, as evidenced in this study by the loss in body mass, both fat and lean, in the $\mathrm{CHO}$ group. In our study, the addition of a BCAA-containing supplement, which has well documented anabolic effects [6], blunted the loss in lean mass in subjects in a hypocaloric state. This anabolic effect of BCAA's has been shown in healthy RT males [7]

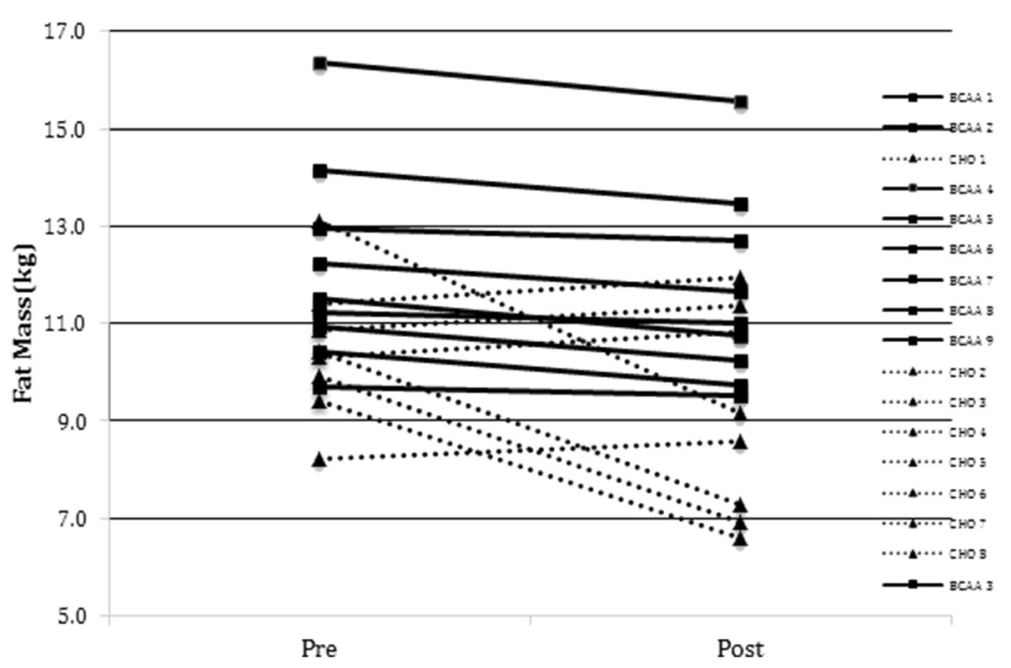

Fig. 2 Individualized changes in lean tissue mass following eight-week study period as determined by hydrostatic weighing. BCAA group received BCAA product (14 g prior/during each workout and $14 \mathrm{~g}$ post workout) while the control group received $28 \mathrm{~g}$ carbohydrate/electrolyte mixture at the same times. All subjects followed an individualized hypocaloric diet and resistance training program 


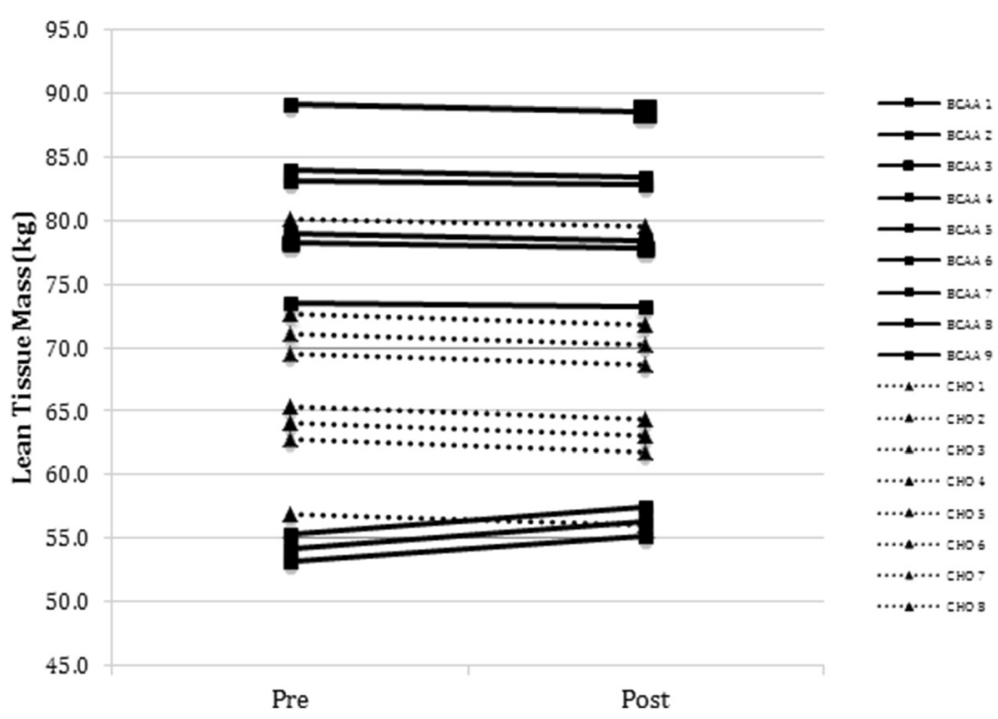

Fig. 3 Individualized changes in fat mass following eight-week study period as determined by hydrostatic weighing. BCAA group received BCAA product (14 g prior/during each workout and $14 \mathrm{~g}$ post workout) while the control group received $28 \mathrm{~g}$ carbohydrate/electrolyte mixture at the same times. All subjects followed an individualized hypocaloric diet and resistance training program

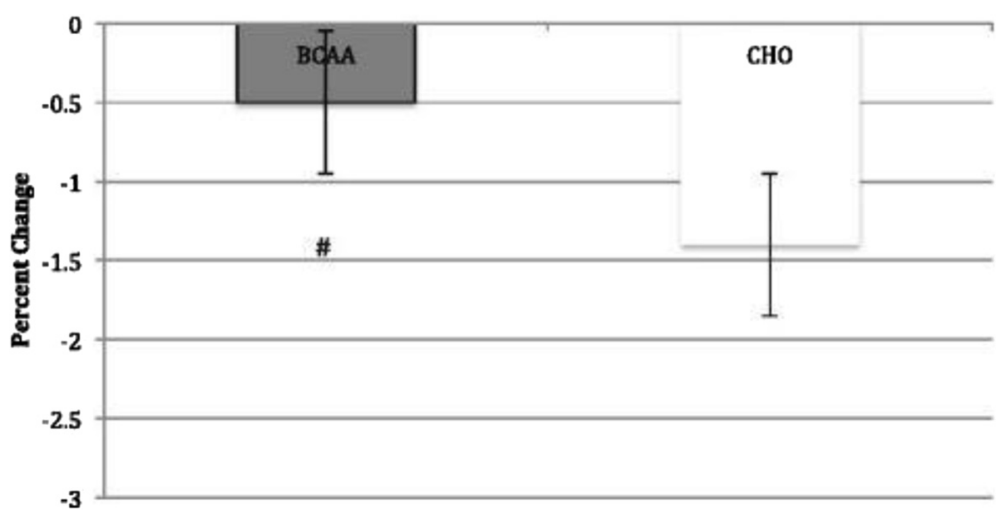

Fig. 4 Change in body mass following eight-week study period as determined by hydrostatic weighing. BCAA group received BCAA product (14 g prior/during each workout and $14 \mathrm{~g}$ post workout) while the control group received $28 \mathrm{~g}$ carbohydrate/electrolyte mixture at the same times. All subjects followed an individualized hypocaloric diet and resistance training program. \# denotes significant difference $(p<0.05)$ within $\mathrm{BCAA}$ and $\mathrm{CHO}$

and in persons attempting to lose weight $[8,9]$, but scarce data exits pertaining to hypocaloric diets.

Is this the seminal study on amino acid supplementation, body mass and resistance training?. By no means. However, what this study does is add to the knowledge base and present other research scientists the opportunity to mimic or expand on our methodology and results to determine what the data collectively says about this specific question. As noted in our acknowledgements, performing heavy resistance exercise while following a strict hypocaloric diet is difficult, to say the least. Thus, there is a paucity of peer-reviewed work looking at reduced caloric intake, amino acid supplementation, body composition and muscle performance.

\section{Author details}

'Providence Medical Research Center, Providence Sacred Heart Medical Center and Children's Hospital, Research Discovery Lab, Spokane, WA, USA.

${ }^{2}$ Department of Health Sciences, Lehman College, Bronx NY, USA. ${ }^{3}$ AARR,

Northridge, CA, USA.

Received: 17 February 2016 Accepted: 6 April 2016

Published online: 11 May 2016

\section{References}

1. Dudgeon WD, Kelley EP, Scheett TP. In a single-blind, matched group design: branched-chain amino acid supplementation and resistance training 
maintains lean body mass during a caloric restricted diet. J Int Soc Sports Nutr. 2016;13:1-015-0112-9. eCollection 2016.

2. Kao LS, Green CE. Analysis of variance: is there a difference in means and what does it mean? J Surg Res. 2008;144(1):158-70.

3. Hopkins WG, Marshall SW, Batterham AM, Hanin J. Progressive statistics for studies in sports medicine and exercise science. Med Sci Sports Exerc. 2009; 41(1):3-13.

4. Nakagawa S, Cuthill IC. Effect size, confidence interval and statistical significance: a practical guide for biologists. Biol Rev Camb Philos Soc. 2007; 82(4):591-605.

5. Morton RW, McGlory C, Phillips SM. Nutritional interventions to augment resistance training-induced skeletal muscle hypertrophy. Front Physiol. 2015; 6:245.

6. Brestenský M, Nitrayová S, Patrás P, Heger J, Nitray J. Branched chain amino acids and their importance in nutrition. J Microbiology Biotech Food Sci. 2015;5(2):197-202.

7. Kreipke VC, Allman BR, Kinsey AW, Moffatt RJ, Hickner RC, Ormsbee MJ. Impact of four weeks of a multi-ingredient performance supplement on muscular strength, body composition, and anabolic hormones in resistancetrained young men.

8. Jitomir J, Willoughby DS. Leucine for retention of lean mass on a hypocaloric diet. J Med Food. 2015;29(12):3453-65.

9. Mettler $S$, Mitchell N, Tipton KD. Increased protein intake reduces lean body mass loss during weight loss in athletes. Med Sci Sport Exer. 2010;42(2):326-37.

Submit your next manuscript to BioMed Central and we will help you at every step:

- We accept pre-submission inquiries

- Our selector tool helps you to find the most relevant journal

- We provide round the clock customer support

- Convenient online submission

- Thorough peer review

- Inclusion in PubMed and all major indexing services

- Maximum visibility for your research

Submit your manuscript at www.biomedcentral.com/submit
C Biomed Central 